\title{
Subcutaneous Administration of PDGF-AA Improves the Functional Recovery After Spinal Cord Injury
}

\author{
Xue-Yan Guo ${ }^{1,2+}$, Fei-Xiang Duan 1,2t, Jing Chen 1,2, Ying Wang ${ }^{1,2}$, Rui Wang ${ }^{2}$, Lin Shen², \\ Qi Qi ${ }^{2}$, Zhi-Quan Jiang ${ }^{2}$, An-You Zhu ${ }^{1}$, Jin Xi' ${ }^{2}$, He-Zuo Lü ${ }^{1,2 *}$ and Jian-Guo Hu ${ }^{1,2 *}$ \\ ${ }^{1}$ Department of Clinical Laboratory, The First Affiliated Hospital of Bengbu Medical College, Bengbu, China, ${ }^{2}$ Anhui Key \\ Laboratory of Tissue Transplantation, Bengbu Medical College, Bengbu, China
}

\section{OPEN ACCESS}

Edited by:

Antonio Oliviero,

Fundación del Hospital Nacional

de Parapléjicos, Spain

Reviewed by:

YeXiong,

Henry Ford Health System,

United States

Marin Manuel,

Université Paris Descartes, France

*Correspondence:

Jian-Guo Hu

jghu9200@163.com

He-Zuo Lü

Ihz233003@163.com

${ }^{\dagger}$ These authors have contributed equally to this work

Specialty section: This article was submitted to

Neurodegeneration,

a section of the journal

Frontiers in Neuroscience

Received: 21 November 2018 Accepted: 07 January 2019

Published: 22 January 2019

Citation:

Guo $X-Y$, Duan F-X, Chen J, Wang $Y$, Wang $R$, Shen $L, Q i Q$, Jiang Z-Q, Zhu A-Y, Xi J, Lü H-Z and

Hu J-G (2019) Subcutaneous Administration of PDGF-AA Improves the Functional Recovery After Spinal

Cord Injury. Front. Neurosci. 13:6.

doi: 10.3389/fnins.2019.00006
Previous studies by our group have demonstrated that the transplantation of exogenous platelet-derived growth factor (PDGF)-AA-overexpressing oligodendrocyte progenitor cells (OPCs) promotes tissue repair and recovery of neurological function in a rat model of spinal cord injury (SCI). However, it remains unclear whether treatment with PDGF-AA also affects endogenous oligodendrocytes (OLs) or even neurons, thus promoting further functional recovery after SCl. In the present study, we evaluated the therapeutic potential of PDGF-AA treatment by direct subcutaneous injection of PDGF-AA immediately after SCl. We demonstrated that PDGF-AA injection resulted in increased tissue sparing, myelination and functional recovery in rats following $\mathrm{SCl}$. Further experimentation confirmed that PDGF-AA increased the survival of endogenous OPCs and OLs, and promoted the proliferation of OPCs and their differentiation into OLs. Moreover, PDGF-AA also protected motor neurons from death in the injured spinal cord. These results indicated that PDGF-AA administration may be an effective treatment for $\mathrm{SCl}$.

\footnotetext{
Keywords: platelet-derived growth factor-AA, subcutaneous administration, spinal cord injury, functional recovery, oligodendrocytes
}

\section{INTRODUCTION}

Spinal cord injury (SCI) is a devastating traumatic neurological disorder, resulting in permanent neurological deficits with limited prospects for spontaneous recovery (Quencer and Bunge, 1996; Ahuja et al., 2017). Recent statistical data showed that the estimated annual incidence of SCI was 54 cases per 1 million people in the United States in 2012 (Jain et al., 2015), an injury for which there are currently no effective treatments. SCI results in the death of oligodendrocytes (OLs) and neurons, leading to demyelination which further impairs neurological function (Liu et al., 1997; McTigue and Tripathi, 2008). Different strategies to promote endogenous repair and replace lost cells have been investigated for SCI in recent years (Thuret et al., 2006; Mothe and Tator, 2012; Silva et al., 2014; Assinck et al., 2017).

A previous study from our group reported that the co-transplantation of Schwann cells (SCs) and oligodendrocyte progenitor cells (OPCs) promoted the survival, proliferation, and migration of transplanted OPCs in vivo, and improved neurological recovery following SCI (Hu et al., 2013). A subsequent in vitro study found that SCs promote the proliferation and migration of 
OPCs by secreting platelet-derived growth factor (PDGF)AA and fibroblast growth factor (FGF)-2 (Chen et al., 2015); moreover, we confirmed in an earlier study that PDGF-AA stimulated the proliferation of OPCs and their differentiation into OLs (Hu et al., 2008). Next, we showed that the transplanted PDGF-AA-overexpressing OPCs promoted tissue repair and recovery of neurological function in a rat model of SCI (Yao et al., 2017). However, it remains unclear whether treatment with PDGF-AA also affects endogenous OLs or even neurons, thus promoting further functional recovery after SCI.

In the present study, we evaluated the therapeutic potential of PDGF-AA administration by subcutaneous administration immediately after SCI. We demonstrated that injection of PDGFAA promoted the proliferation of endogenous OPCs and their differentiation into OLs, increased the survival of OPC, OLs and motor neurons in the injured rat spinal cord, resulting in increased tissue sparing, myelination, and functional recovery post-SCI.

\section{MATERIALS AND METHODS}

\section{Animals}

Adult (2 months old) female Sprague-Dawley rats (weighing 200-220 g) were used. All procedures were approved by the Animal Care and Use Committees of Bengbu Medical College, and were in accordance with the Guide for the Care and Use of Laboratory Animals (National Research Council, 1996) and the Guidelines and Policies for Rodent Survival Surgery, provided by the Animal Care and Use Committees of Bengbu Medical College. In total 32 rats were used in the study.

\section{Contusive SCI and PDGF-AA Administration}

Contusive SCI was performed with the weight-drop method using a New York University Impactor (Gruner, 1992;

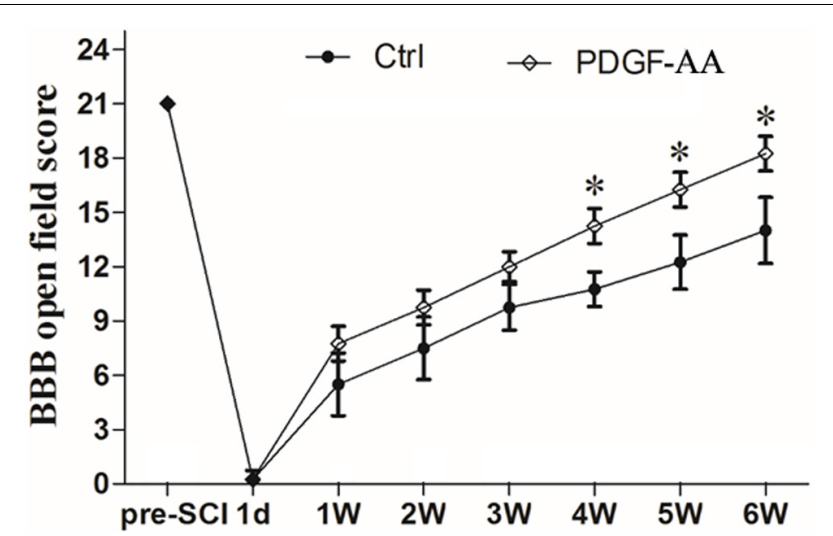

FIGURE 1 | Treatment with PDGF-AA improves locomotor functional recovery after SCI. Spinal cord-injured rats that received treatment with PDGF-AA showed greater improvement in locomotor BBB scores at 4-6 weeks post-SCl, as compared to controls $(n=8)$. ${ }^{*} P<0.05$.
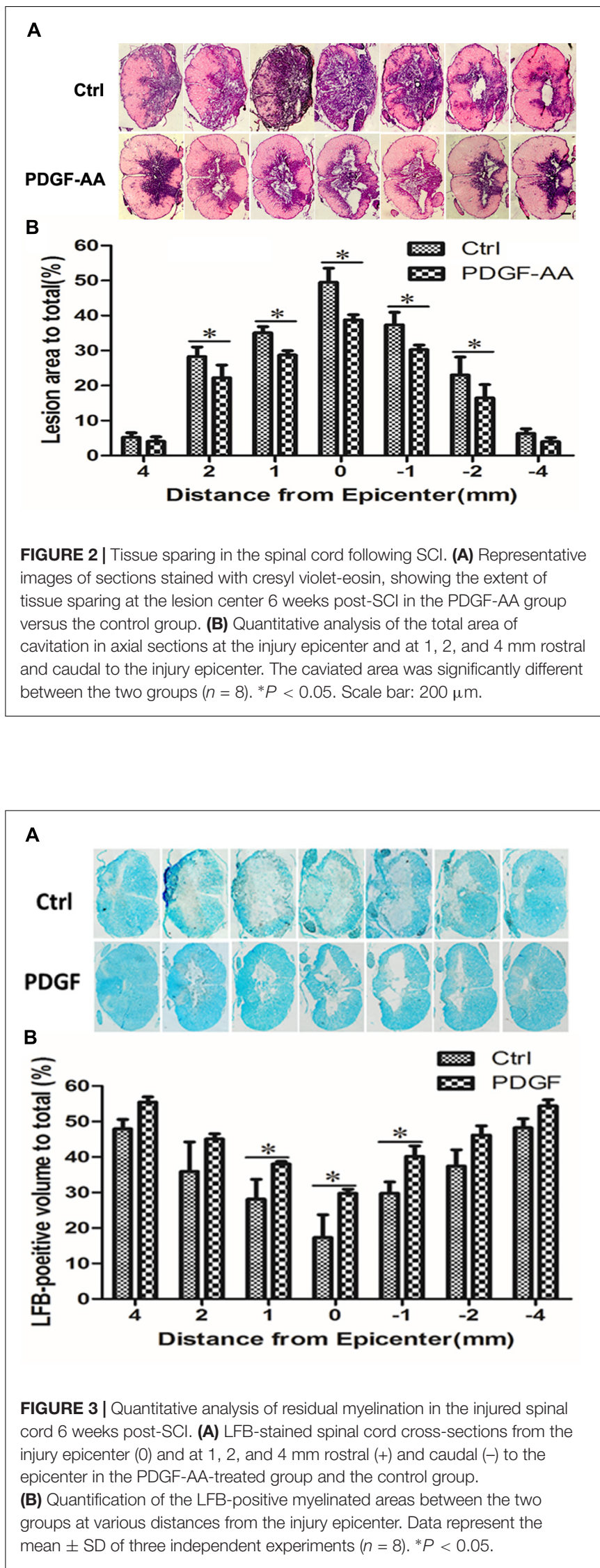
A

Ctrl

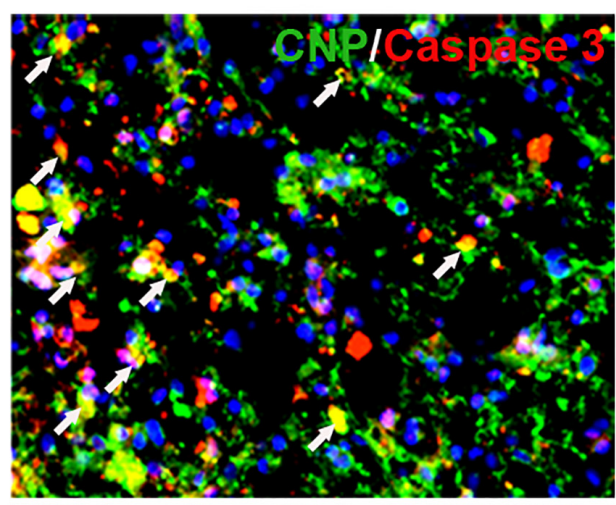

B

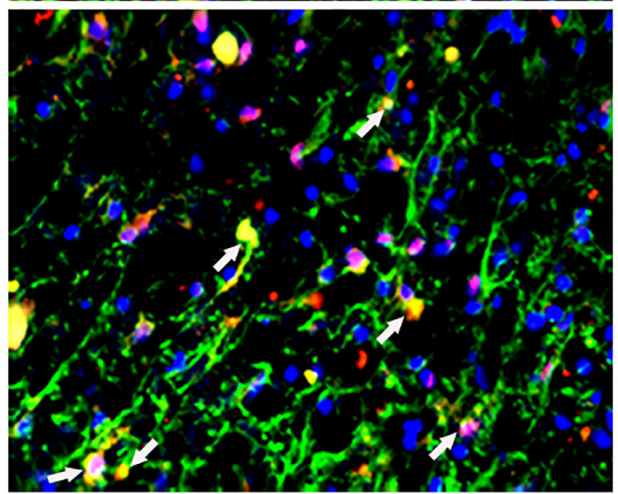

C

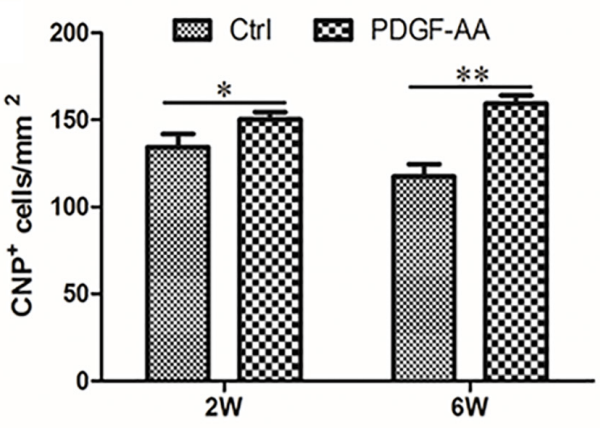

PDGF-AA
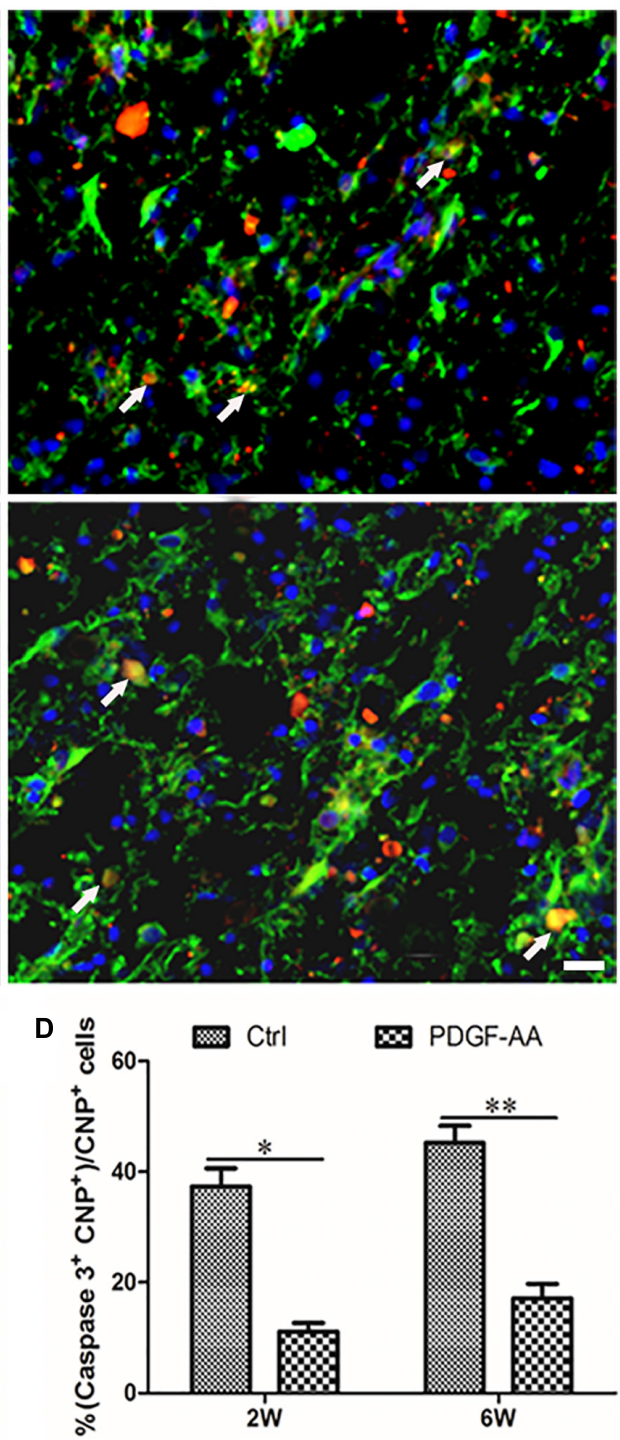

FIGURE 4 | Proliferation of OPCs in the injured spinal cord post-SCl, as determined by BrdU incorporation. (A,B) Representative photomicrographs showing BrdU+ cells (green) co-localized with O4+OPCs (red) in the circumambience of the injured spinal cord of rats at 2 and 6 weeks post-SCl. (C,D) Quantitative analysis of OPC proliferation, demonstrating an increase in both the number and percentage of BrdU+OPCs in rats that received PDGF-AA versus controls. Data represent the mean $\pm \mathrm{SD}(n=8) .{ }^{*} P<0.05 ; * * P<0.01$. Scale bar $=20 \mu \mathrm{m}$.

Hu et al., 2013). Briefly, rats were anesthetized with pentobarbital $(50 \mathrm{mg} / \mathrm{kg}$ ) dissolved in phosphate buffered saline (PBS), injected intraperitoneally. Rats then received a laminectomy at the T10 level, and the dorsal surface of the spinal cord was subjected to a weight-drop impact of $10 \mathrm{~g}$, dropped from a height of $12.5 \mathrm{~mm}$. After SCI, rats were randomly assigned to the controlPBS or PDGF-AA injection group. PBS or human PDGF-AA (Invitrogen, Carlsbad, CA, United States; $300 \mu \mathrm{g} / \mathrm{kg}$ body weight each time based on our pretest) was subcutaneously injected into the back skin of rats at $30 \mathrm{~min}$ and every second day post-SCI for 2 weeks. The muscle and skin were sutured layer by layer and then sterilized with iodine. Rats were placed in a temperature- and humidity-controlled chamber, and post-operative monitoring included manual bladder emptying three times a day until reflexive bladder control was re-established. Moreover, rats received an analgesic agent (buprenorphine, $0.3 \mathrm{mg} / \mathrm{kg}$ ) twice a day for 3 days to alleviate pain. To prevent infection, animals were provided with chloramphenicol $(50-75 \mathrm{mg} / \mathrm{kg})$ daily via the drinking water. Subsequently, animals were sacrificed at 2 or 6 weeks post-SCI.

\section{Behavioral Assessment}

Open-field locomotor testing was carried out by two trained investigators using the 21-point Basso, Bresnahan, and Beattie 
A

B

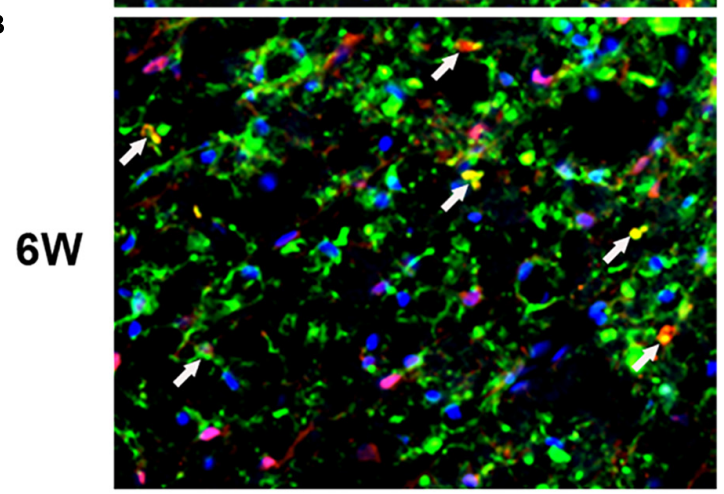

C

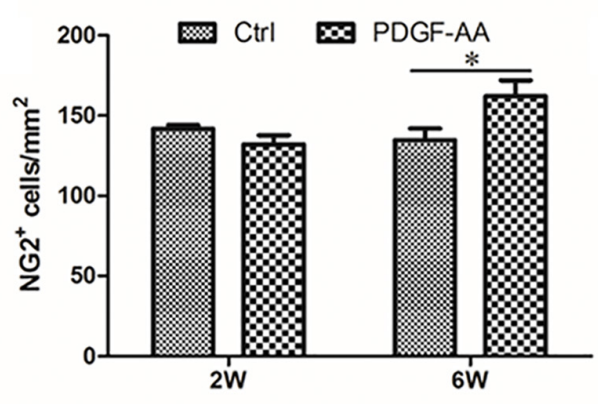

Ctrl

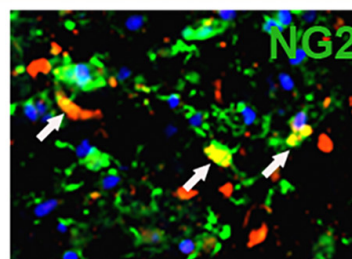

2W

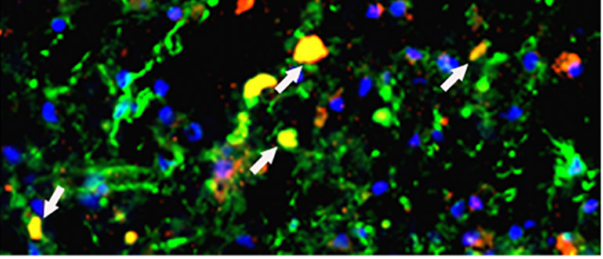

PDGF-AA
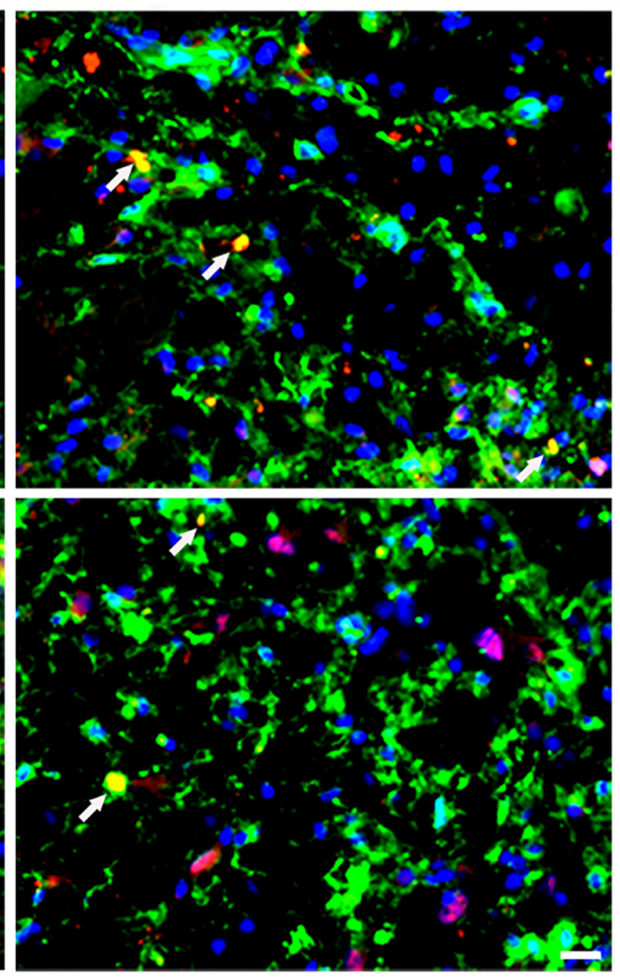

D

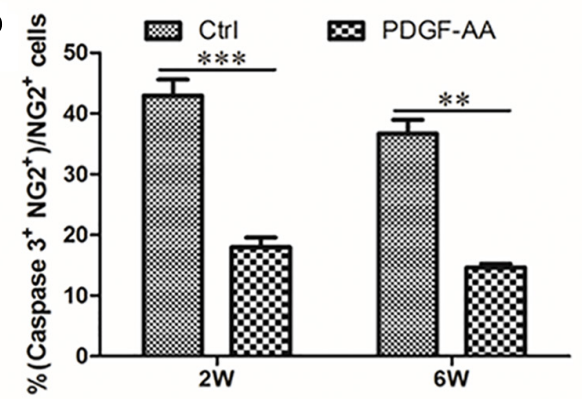

FIGURE 5 | Differentiation of OLs from proliferated OPCs in the injured spinal cord after SCl, as determined by BrdU incorporation. (A,B) Representative photomicrographs showing BrdU+cells (green) co-localized with CNP+OLs (red) in the circumambience of the injured spinal cord of rats at 2 and 6 weeks post-SCl. (C,D) Quantitative analysis of BrdU+CNP+OLs showing that both the number and percentage of BrdU+OLs were higher in rats that received PDGF-AA versus controls. Data represent the mean $\pm \operatorname{SD}(n=8)$. ${ }^{*} P<0.05 ;{ }^{* *} P<0.01 ;{ }^{* * *} P<0.001$. Scale bar $=20 \mu \mathrm{m}$.

(BBB) locomotor scale (Basso et al., 1995) once a week postinjury to assess hindlimb locomotor recovery, including joint movement, stepping ability, coordination, and trunk stability.

\section{Tissue Preparation}

At predetermined time points ( 2 and 6 weeks post-injury), rats were anesthetized with $60 \mathrm{mg} / \mathrm{kg}$ pentobarbital and transcardially perfused with $4 \%$ paraformaldehyde (PFA) in $0.01 \mathrm{M}$ PBS, $\mathrm{pH}$ 7.4. Spinal cord segments containing the injury site were removed, post-fixed in the same fixative overnight at $4^{\circ} \mathrm{C}$, and cryoprotected in 30\% sucrose (Sigma-Aldrich, St. Louis, MO, United States) buffer for 5-7 days. A $2 \mathrm{~cm}$ length of the spinal cord, centered at the injection or injury site, was dissected and embedded in HistoPrep (Fisher Scientific, Pittsburgh, PA, United States) on dry ice. After the spinal cords were mounted in blocks, serial $20 \mu \mathrm{m}$-thick sections through the entire injury site were cut on a cryostat. All spinal cords from each group ( $n=8 \mathrm{rats} /$ group) were cut as transverse sections. Sections were mounted on gelatin-coated slides (Fisher Scientific, Waltham, MA, United States), and stored at $-70^{\circ} \mathrm{C}$.

\section{Histological Analysis}

Two sets of transverse sections (each set containing serial sections spaced $500 \mu \mathrm{m}$ apart) from rat spinal cord at 6 weeks postSCI were stained with cresyl violet-eosin (Sigma) for lesion area assessment ( $n=8$ /group) and Luxol Fast Blue (LFB; Sigma) 

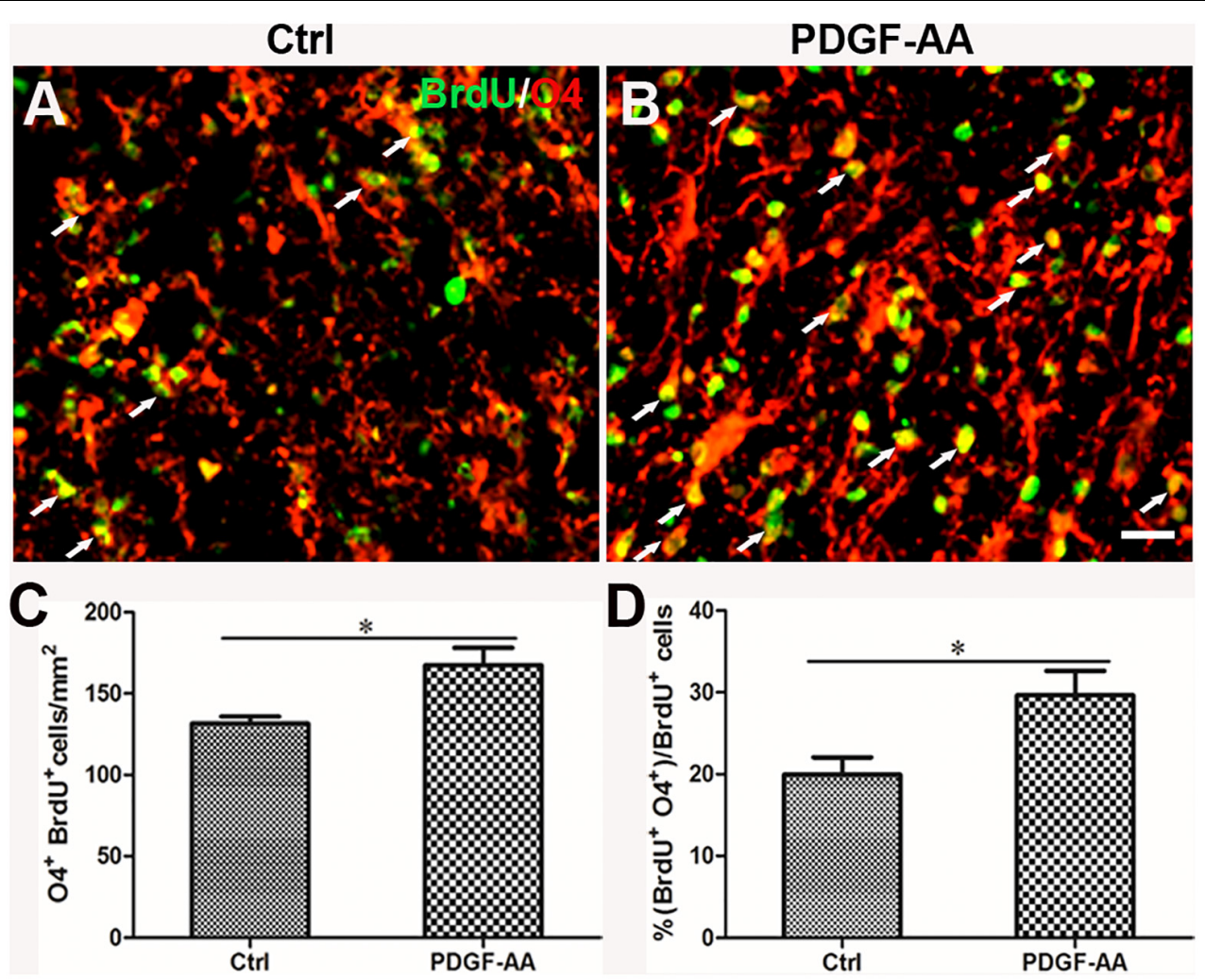

FIGURE 6 | Survival of OLs in the injured spinal cord after SCI, as determined by activated caspase-3 staining. (A,B) Representative photomicrographs showing caspase-3+cells (red) co-localized with CNP+OLs (green) in the circumambience of the injured spinal cord of rats at 2 weeks post-SCl. (C,D) Quantitative analysis of OL survival showing that both the number and percentage of caspase-3+OLs were higher in rats that received PDGF-AA versus controls. Data represent the mean $\pm \mathrm{SD}(n=8)$. $* P<0.05$. Scale bar $=20 \mu \mathrm{m}$.

for white matter sparing analysis ( $n=8$ /group), as previously described (Hu et al., 2013). The total area of cavitation and LFBpositive myelinated areas in axial sections at the injury epicenter and at 1,2, and $4 \mathrm{~mm}$ rostral and caudal to the injury epicenter were outlined and measured using the Neurolucida System (MicroBrightField, Colchester, VT, United States) connected to a BX60 microscope (Olympus, Tokyo, Japan), and are expressed as a percentage of the total stained area. The lesion center was defined as the section containing the least amount of spared white matter.

\section{Immunohistochemistry}

Cryostat sections were stained using standard immunohistochemistry techniques. Frozen sections collected on slides were air-dried at room temperature for $10 \mathrm{~min}$ and washed with PBS for $10 \mathrm{~min}$, then blocked with Tris-buffered saline containing $10 \%$ donkey serum and $0.3 \%$ Triton X-100 for $1 \mathrm{~h}$ at room temperature. Primary antibodies in the same blocking solution were applied overnight at $4^{\circ} \mathrm{C}$. The primary antibodies used were as follows: rabbit anti-caspase- 3 antibody (1:200; Abcam, Cambridge, MA, United States), mouse anti-NG2 (1:200; Abcam), mouse anti-beta III tubulin (1:200; Abcam), and mouse anti-CNPase (CNP; 1:200; Abcam). The slides were then washed three times in PBS and incubated with fluorescein isothiocyanate (FITC)- or rhodamine-conjugated donkey antirabbit or mouse IgG (all at 1:200; Jackson Laboratores, West Grove, PA, United States) for $1 \mathrm{~h}$ at $37^{\circ} \mathrm{C}$. Slides were washed three times with PBS and mounted with Gel/Mount containing Hoechst 33342 to counterstain the nuclei. Images were acquired with a Zeiss Axio Observer fluorescence microscope. Control samples were prepared by omitting the primary antibody. The spinal cord cross-sections from six rats per timepoint were used for evaluation. Five complete sections per animal were analyzed in a blinded fashion, and cell numbers were calculated as the number of cells in a set of five slides, from rostral to caudal, containing the injury epicenter.

\section{Bromodeoxyuridine (BrdU) Incorporation Assay}

To assess cell proliferation after PDGF-AA administration, 3 days prior to the end of the 2 -week post-SCI timepoint, randomly selected rats ( $n=8$ rats/group) were given 10 intraperitoneal injections of $\mathrm{BrdU}(50 \mathrm{mg} / \mathrm{kg} /$ injection; three times daily for 3 days, and one injection on the last day). Rats were sacrificed $2 \mathrm{~h}$ after the last injection. The animals were perfused, their spinal cords were dissected, and the tissue was sectioned. One set of serial sections was randomly selected for the BrdU incorporation assay. Fixed sections were treated with $1 \mathrm{~N} \mathrm{HCl}$ for $40 \mathrm{~min}$ 

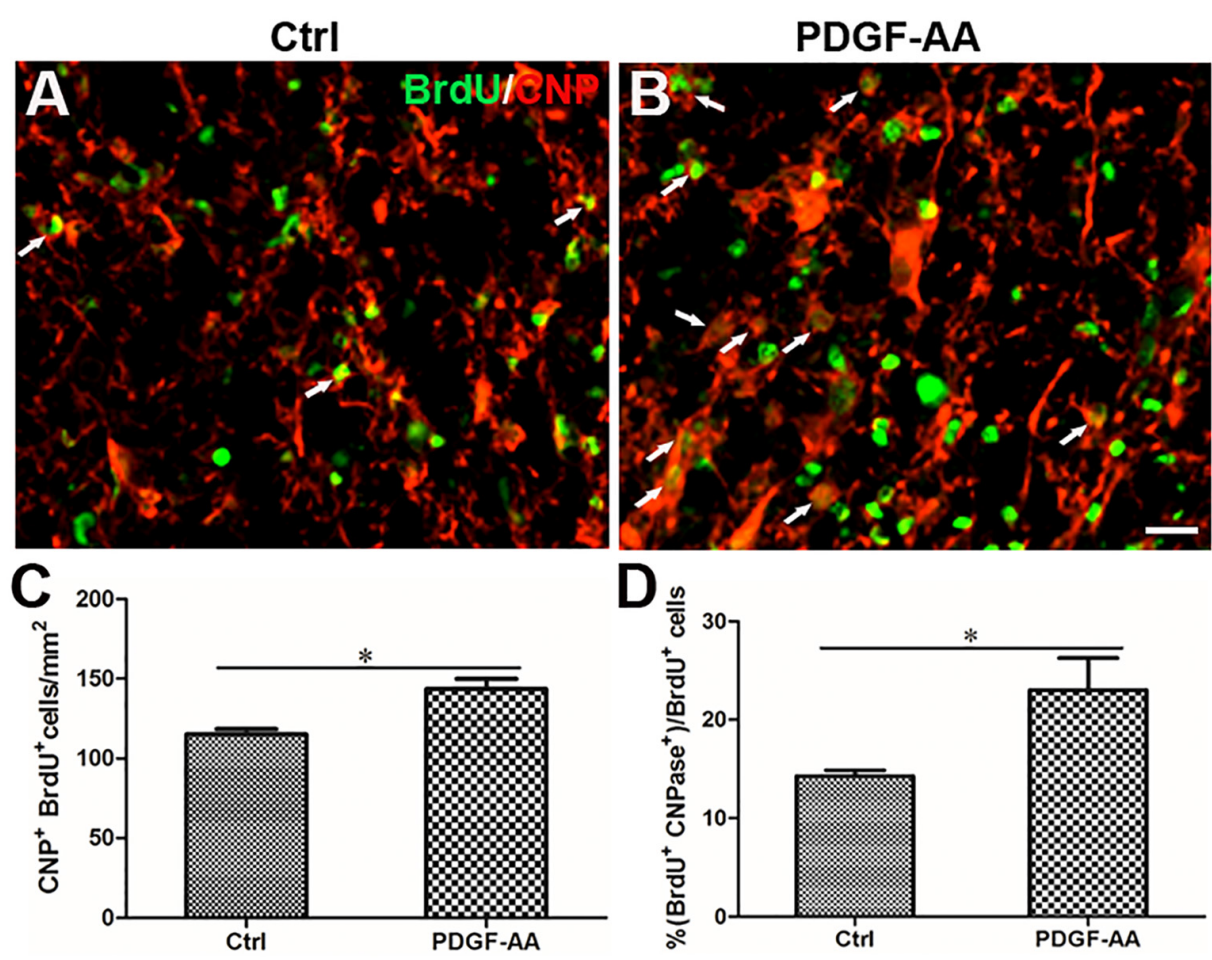

FIGURE 7 | Survival of OPCs in the injured spinal cord after SCl, as determined by activated caspase-3 staining. (A,B) Representative photomicrographs showing caspase-3+cells (red) co-localized with NG2+OPCs (green) in the circumambience of the injured spinal cord of rats at 2 weeks post-SCl. (C,D) Quantitative analysis of OPC survival showed that both the number and percentage of caspase-3+ OPCs were higher in rats that received PDGF-AA versus controls. Data represent the mean $\pm \mathrm{SD}(n=8)$. ${ }^{*}>0.05$. Scale bar $=20 \mu \mathrm{m}$.

at $37^{\circ} \mathrm{C}$ to denature the DNA. The rabbit anti-BrdU antibody (1:100; Sigma-Aldrich) and the mouse anti-O4 (1:100; Chemicon, Temecula, CA, United States), or CNP (1:200; Abcam) antibodies were applied overnight at $4^{\circ} \mathrm{C}$, followed by FITC-conjugated donkey anti-rabbit IgG (1:200; Jackson Labs) and rhodamineconjugated donkey anti-mouse IgM (1:200; Jackson Labs) or IgG (1:200; Jackson Labs) as secondary antibodies at room temperature for $2 \mathrm{~h}$. Slides were washed three times with PBS and mounted with Gel/Mount. Images were acquired with a Zeiss Axio Observer fluorescence microscope. At least five randomly selected fields in each section with a total of more than $500 \mathrm{O} 4+$ or $\mathrm{CNP}+$ cells were counted. The percentage of $\mathrm{O} 4+/ \mathrm{BrdU}+$ or $\mathrm{CNP}+/ \mathrm{BrdU}+$ cells out of the total number of $\mathrm{O} 4+$ or $\mathrm{CNP}+$ cells was determined.

\section{Statistical Analysis}

Data are presented as the mean \pm standard deviation (SD). A one-way analysis of variance (ANOVA) with a Tukey's or Fisher's post hoc test was used to evaluate mean differences. BBB scores were analyzed using a repeated measures ANOVA, followed by a Tukey's pairwise comparison at each timepoint. Other data were analyzed using a non-parametric Kruskal-Wallis ANOVA, followed by individual Mann-Whitney $U$-tests. A $P$ value $<0.05$ was considered statistically significant. Data were analyzed using SPSS v.14.0 software (SPSS, Inc., Chicago, IL, United States).

\section{RESULTS}

\section{Treatment With PDGF-AA Promotes Functional Recovery After SCI}

In order to examine whether subcutaneous PDGF-AA injections could promote functional recovery after SCI, BBB scoring was performed. One day after SCI, all injured rats were paraplegic with no observable hind limb movement. In the first 3 weeks after injection of PDGF-AA, there was no difference between the control and PDGF-AA-treated groups in terms of BBB score (Figure 1). However, with continued recovery, rats that were administered PDGF-AA showed a significant improvement in their BBB score, as compared to the control group, at 4-6 weeks post-injection ( $P<0.05, n=8$; Figure 1$)$. Thus, the PDGF-AA injection regime resulted in improved functional recovery.

\section{Treatment With PDGF-AA Increases Tissue Sparing in the Injured Spinal Cord}

$\mathrm{H} \& \mathrm{E}$ staining was performed in order to examine histological morphology of the injury site at 6 weeks post-SCI. Consistent with the locomotion evaluation, the PDGF-AA-treated group showed less tissue damage, with a smaller lesion area compared to the control group $(P<0.05, n=8$; Figures $2 A, \mathbf{B})$, indicating that treatment with PDGF-AA increased tissue sparing in the injured 


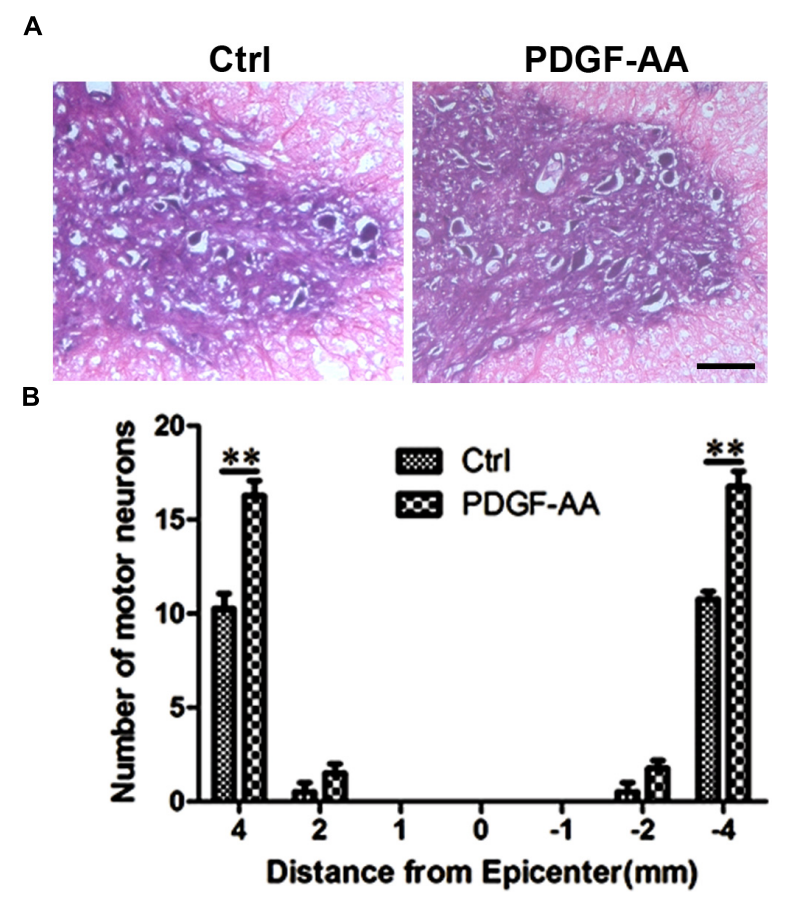

FIGURE 8 | Motor neuron survival in the ventral horn of the spinal cord 6 weeks post-SCI. (A) Representative images of the ventral horn $4 \mathrm{~mm}$ rostral to the injury epicenter in the control and PDGF-AA-treated groups.

(B) Comparison of the numbers of ventral horn neurons in different groups at various distances from the injury epicenter (0) and at 1, 2, and $4 \mathrm{~mm}$ rostral (+) and caudal ( - ) to the epicenter. Data represent the mean \pm SD of three independent experiments $(n=8)$. ${ }^{* *} P<0.01$. Scale bar $=200 \mu \mathrm{m}$.

spinal cord, and ameliorated the pathological morphology of the lesion area.

\section{Treatment With PDGF-AA Increases Myelination in the Injured Spinal Cord}

To investigate whether administration of PDGF-AA improved the preservation of existing myelin and/or promotes remyelination, LFB staining was performed to assess the extent of myelination at the injury center 6 weeks post-PDGF-AA injection. We observed that the area of LFB staining at the injury center was larger in rats treated with PDGF-AA, compared to the control group that did not receive PDGF-AA $(P<0.05, n=8$; Figures 3A,B).

\section{Treatment With PDGF-AA Promotes the Survival of OLs and OPCs in the Injured Spinal Cord}

Activated caspase- 3 staining revealed that at both 2 and 6 weeks there were more CNP+ OLs (112.75 \pm 3.11 versus $98 \pm 2.83 \%$ at 2 weeks, $P<0.05, n=8$, Figures 4A,C; $121 \pm 0.71$ versus $88 \pm 5.48 \%$ at 6 weeks, $P<0.05, n=8$, Figures 4B,C) and fewer caspase-3+/CNP+ OLs $(11.12 \pm 1.54$ versus $37.33 \pm 3.31 \%$ at 2 weeks, $P<0.05, n=8$, Figures 4A,D; $17.14 \pm 2.59$ versus $45.23 \pm 3.07 \%$ at 6 weeks, $\mathrm{P}<0.05, n=8$, Figures 4B,D) at the periphery of the injury center in rats treated with PDGF-AA, as compared to control rats that did not receive PDGF-AA. Similarly, more NG2+ OPCs (121.5 \pm 7.40 versus $101.12 \pm 5.39 \%$ at 6 weeks, $P<0.05, n=8$, Figures 5B,C) and fewer caspase-3+/NG2+ OPCs (17.97 \pm 1.58 versus $42.95 \pm 2.66 \%$ at 2 weeks, $P<0.05, n=8$, Figures 5A,D; $14.60 \pm 0.62$ versus $36.67 \pm 2.32 \%$ at 6 weeks, $P<0.05, n=8$, Figures 5B,D) at the periphery of the injury center were observed in rats treated with PDGF-AA as compared to control rats that did not receive PDGF-AA.

\section{Treatment With PDGF-AA Promotes the Proliferation of OPCs and Their Differentiation Into OLs in the Injured Spinal Cord}

A BrdU incorporation assay at 2 weeks post-SCI revealed an increase in the number of both BrdU+/O4+ OPCs $(167.33 \pm 9.31$ versus $131.56 \pm 3.50 \%, P<0.05, n=8$, Figures 6A-C) and BrdU+/CNP+ OLs (143.67 \pm 5.45 versus $115.11 \pm 2.74 \%, P<0.05, n=8$, Figures $7 \mathrm{~A}-\mathrm{C})$ at the injury center in rats treated with PDGF-AA as compared to control rats that did not receive PDGF-AA. Similarly, the percentages of BrdU+ cells among both $\mathrm{O} 4+$ cells (29.52 \pm 2.76 versus $19.95 \pm 1.71 \%, P<0.05, n=8$, Figures 6A,B,D) and CNP+ cells $(23.00 \pm 2.82$ versus $14.26 \pm 0.47 \%, P<0.05, n=8$, Figures 7A,B,D) at the injury center were greater in rats treated with PDGF-AA as compared to controls that did not receive PDGF-AA. These data confirmed that treatment with PDGFAA promoted the proliferation of endogenous OPCs and their differentiation into OLs in the injured spinal cord post-SCI.

\section{Treatment With PDGF-AA Promotes the Survival of Motor Neurons in the Ventral Horn Following SCI}

To determine the effect of PDGF-AA administration on motor neuronal survival, the number of ventral horn motor neurons at the injury epicenter and at 1,2, and $4 \mathrm{~mm}$ rostral and caudal to the epicenter were counted 6 weeks post-SCI. An increase in the number of residual motor neurons was observed in the ventral horn $4 \mathrm{~mm}$ rostral and caudal to the lesion epicenter in the PDGF-AA-treated group, as compared to the control group $(P<0.01, n=8$, Figure 8).

\section{DISCUSSION}

We previously found that co-transplantation of SCs enhanced functional recovery in a rat model of SCI (Hu et al., 2013) via secretion of PDGF-AA and FGF-2 (Chen et al., 2015). Subsequently, we showed that the transplantation of PDGFAA-overexpressing OPCs promoted tissue repair and recovery of neurological function after SCI (Yao et al., 2017). In the present study, the spinal cord injured rats received subcutaneous administration of PDGF-AA to investigate whether it effects endogenous OLs and other neural cells to lead to a therapeutic effect. 
In this study, we found no difference in $\mathrm{BBB}$ score during the first 3 weeks post-SCI in rats with or without PDGF-AA administration. However, at 4-6 weeks post-SCI, we observed a significant improvement in the $\mathrm{BBB}$ score in rats that received PDGF-AA injections, as compared to control rats that did not receive PDGF-AA injections. This behavioral finding was consistent with our previous results of OPC/SC cotransplantation (Hu et al., 2013), as well as results from the transplantation of PDGF-AA-overexpressing OPCs (Yao et al., 2017). This result suggested that PDGF-AA administration was able to promote functional locomotor recovery after $\mathrm{SCI}$ in rats.

We next evaluated whether PDGF-AA injection promoted tissue repair in the injured spinal cord. We observed a significant reduction in the spinal cord lesion area in rats that received PDGF-AA injections compared to control rats, indicating that PDGF-AA injection enhanced tissue repair following SCI, which explained the functional improvement observed in these animals.

Preservation of existing myelin and re-myelination is the basis of functional recovery following SCI (Keirstead et al., 2005; $\mathrm{Xu}$ and Onifer, 2009). In our study, there was more residual myelin in rats that received PDGF-AA injection as compared to control rats at 6 weeks post-SCI, indicating that treatment with PDGF-AA preserved residual myelin and promoted the re-myelination of demyelinated axons in the injured spinal cord.

Spinal cord injury leads to the death of a massive number of cells, including neurons and OLs that myelinate the axons of surviving neurons (Casha et al., 2001; Beattie et al., 2002; Stenudd et al., 2015). It has been reported that PDGF, in synergy with bFGF, regulates the proliferative response of adult OPCs (Lachapelle et al., 2002; Frost et al., 2003; KarimiAbdolrezaee et al., 2012). Our previous study also confirmed that overexpression of PDGF-AA promoted the proliferation and survival of transplanted OPCs (Yao et al., 2017), which resulted in increased myelination and tissue repair, leading to improved functional recovery. To investigate the mechanism by which PDGF-AA treatment promoted functional recovery and tissue repair following SCI, we examined the effect of PDGF-AA treatment on the survival and proliferation of endogenous OLs around the injury site in the spinal cord following SCI. Activated caspase-3 labeling and BrdU incorporation revealed that PDGF-AA administration protected both OPCs and OLs from apoptosis, and promoted the proliferation of OPCs and their differentiation into OLs after SCI. These results suggested that PDGF-AA treatment increased the number of myelin-forming cells at the injury site post-SCI, which led to further myelin repair and functional recovery following SCI.

\section{REFERENCES}

Ahuja, C. S., Wilson, J. R., Nori, S., Kotter, M. R. N., Druschel, C., Curt, A., et al. (2017). Traumatic spinal cord injury. Nat. Rev. Dis. Primers 3:17018. doi: $10.1038 / \mathrm{nrdp} .2017 .18$

Assinck, P., Duncan, G. J., Hilton, B. J., Plemel, J. R., and Tetzlaff, W. (2017). Cell transplantation therapy for spinal cord injury. Nat. Neurosci. 20, 637-647. doi: $10.1038 / \mathrm{nn} .4541$
The death of neurons is another key factor that results in the loss of function after SCI (Siddiqui et al., 2015). It has been reported that PDGF-AA suppresses the $\mathrm{Ca}^{2+}$ overload induced by $\mathrm{H}_{2} \mathrm{O}_{2}$ in mouse cortical neuron primary cultures (Zheng et al., 2013), and protects them from $\mathrm{H}_{2} \mathrm{O}_{2}$-induced oxidative stress (Zheng et al., 2010). Moreover, Cheng and Mattson (1995) found that pretreatment of rat and mouse hippocampal neurons with PDGF-AA resulted in a highly significant attenuation of glucose deprivation- and $\mathrm{FeSO}_{4}$-induced neuronal degeneration. However, it remained unclear whether PDGF-AA injection affected the survival of neurons in the injured spinal cord after SCI. In this study, we observed an increase in the number of residual motor neurons in the ventral horn rostral and caudal to the lesion epicenter in PDGF-AA-treated rats. This result suggested that treatment with PDGF-AA promoted the survival of neurons following SCI. Together with the observed effect of PDGF-AA treatment on the survival of endogenous OPCs and OLs, and the proliferation of OPCs and their differentiation into OLs, our results clarified why PDGF-AA treatment promoted the repair of injured spinal cord tissue, resulting in improved functional recovery after SCI.

\section{CONCLUSION}

Our results demonstrated that PDGF-AA treatment promoted the survival of OLs and neurons and increased the proliferation of endogenous OPCs and their differentiation into OLs postSCI, which increased the amount of myelination and tissue repair in the injured spinal cord, leading to improved recovery of neurological function. These results suggested that treatment with PDGF-AA may be a potential strategy to promote recovery following SCI.

\section{AUTHOR CONTRIBUTIONS}

J-GH and H-ZL designed the experiments and edited the manuscript. X-YG, F-XD, and JC performed the experiments, analyzed the data, and wrote the manuscript. YW, RW, and Z-QJ interpreted the data and prepared the figures. LS, QQ, JX, and A-YZ performed the experiments and analyzed the data.

\section{FUNDING}

This work was supported by the National Natural Science Foundation of China (Grant Nos. 81471277 and 81771343).

Basso, D. M., Beattie, M. S., and Bresnahan, J. C. (1995). A sensitive and reliable locomotor rating scale for open field testing in rats. J. Neurotrauma 12, 1-21. doi: 10.1089/neu.1995.12.1

Beattie, M. S., Hermann, G. E., Rogers, R. C., and Bresnahan, J. C. (2002). Cell death in models of spinal cord injury. Prog. Brain Res. 137, 37-47. doi: 10.1016/S00796123(02)37006-7

Casha, S., Yu, W. R., and Fehlings, M. G. (2001). Oligodendroglial apoptosis occurs along degenerating axons and is associated with FAS and p75 expression 
following spinal cord injury in the rat. Neuroscience. 103, 203-218. doi: 10.1016/ S0306-4522(00)00538-8

Chen, Y. J., Zhang, J. X., Shen, L., Qi, Q., Cheng, X. X., Zhong, Z. R., et al. (2015). Schwann cells induce proliferation and migration of oligodendrocyte precursor cells through secretion of PDGF-AA and FGF-2. J. Mol. Neurosci. 56, 999-1008. doi: 10.1007/s12031-015-0570-1

Cheng, B., and Mattson, M. P. (1995). PDGFs protect hippocampal neurons against energy deprivation and oxidative injury: evidence for induction of antioxidant pathways. J. Neurosci. 15, 7095-7104. doi: 10.1523/JNEUROSCI.15-11-07095. 1995

Frost, E. E., Nielsen, J. A., Le, T. Q., and Armstrong, R. C. (2003). PDGF and FGF2 regulate oligodendrocyte progenitor responses to demyelination. J. Neurobiol. 54, 457-472. doi: 10.1002/neu.10158

Gruner, J. A. (1992). A monitored contusion model of spinal cord injury in the rat. J. Neurotrauma 9, 123-126. doi: 10.1089/neu.1992.9.123

Hu, J. G., Fu, S. L., Wang, Y. X., Li, Y., Jiang, X. Y., Wang, X. F., et al. (2008). Platelet-derived growth factor-AA mediates oligodendrocyte lineage differentiation through activation of extracellular signal-regulated kinase signaling pathway. Neuroscience 151, 138-147. doi: 10.1016/j.neuroscience. 2007.10.050

Hu, J. G., Wang, X. F., Deng, L. X., Liu, N. K., Gao, X., Chen, J. H., et al. (2013). Cotransplantation of glial restricted precursor cells and Schwann cells promotes functional recovery after spinal cord injury. Cell Transplant. 22, 2219-2236. doi: $10.3727 / 096368912 X 661373$

Jain, N. B., Ayers, G. D., Peterson, E. N., Harris, M. B., Morse, L., O'Connor, K. C., et al. (2015). Traumatic spinal cord injury in the United States, 1993-2012. JAMA 313, 2236-2243. doi: 10.1001/jama.2015.6250

Karimi-Abdolrezaee, S., Schut, D., Wang, J., and Fehlings, M. G. (2012). Chondroitinase and growth factors enhance activation and oligodendrocyte differentiation of endogenous neural precursor cells after spinal cord injury. PLoS One 7:e37589. doi: 10.1371/journal.pone.0037589

Keirstead, H. S., Nistor, G., Bernal, G., Totoiu, M., Cloutier, F., Sharp, K., et al. (2005). Human embryonic stem cell-derived oligodendrocyte progenitor cell transplants remyelinate and restore locomotion after spinal cord injury. J. Neurosci. 25, 4694-4705. doi: 10.1523/JNEUROSCI.0311-05.2005

Lachapelle, F., Avellana-Adalid, V., Nait-Oumesmar, B., and Baron-Van Evercooren, A. (2002). Fibroblast growth factor-2 (FGF-2) and platelet-derived growth factor $\mathrm{AB}$ (PDGF $\mathrm{AB}$ ) promote adult SVZ-derived oligodendrogenesis in vivo. Mol. Cell. Neurosci. 20, 390-403. doi: 10.1006/mcne.2002.1124

Liu, X. Z., Xu, X. M., Hu, R., Du, C., Zhang, S. X., McDonald, J. W., et al. (1997). Neuronal and glial apoptosis after traumatic spinal cord injury. J. Neurosci. 17, 5395-5406. doi: 10.1523/JNEUROSCI.17-14-05395.1997

McTigue, D. M., and Tripathi, R. B. (2008). The life, death, and replacement of oligodendrocytes in the adult CNS. J. Neurochem. 107, 1-19. doi: 10.1111/j. 1471-4159.2008.05570.x
Mothe, A. J., and Tator, C. H. (2012). Advances in stem cell therapy for spinal cord injury. J. Clin. Invest. 122, 3824-3834. doi: 10.1172/JCI64124

Quencer, R. M., and Bunge, R. P. (1996). The injured spinal cord: imaging, histopathologic clinical correlates, and basic science approaches to enhancing neural function after spinal cord injury. Spine 21, 2064-2066. doi: 10.1097/ 00007632-199609150-00002

Siddiqui, A. M., Khazaei, M., and Fehlings, M. G. (2015). Translating mechanisms of neuroprotection, regeneration, and repair to treatment of spinal cord injury. Prog. Brain Res. 218, 15-54. doi: 10.1016/bs.pbr.2014.12.007

Silva, N. A., Sousa, N., Reis, R. L., and Salgado, A. J. (2014). From basics to clinical: a comprehensive review on spinal cord injury. Prog. Neurobiol. 114, 25-57. doi: 10.1016/j.pneurobio.2013.11.002

Stenudd, M., Sabelström, H., and Frisén, J. (2015). Role of endogenous neural stem cells in spinal cord injury and repair. JAMA Neurol. 72, 235-237. doi: 10.1001/jamaneurol.2014.2927

Thuret, S., Moon, L. D., and Gage, F. H. (2006). Therapeutic interventions after spinal cord injury. Nat. Rev. Neurosci. 7, 628-643. doi: 10.1038/nrn 1955

Xu, X. M., and Onifer, S. M. (2009). Transplantation-mediated strategies to promote axonal regeneration following spinal cord injury. Respir. Physiol. Neurobiol. 169, 171-182. doi: 10.1016/j.resp.2009.07.016

Yao, Z. F., Wang, Y., Lin, Y. H., Wu, Y., Zhu, A. Y., Wang, R., et al. (2017), Transplantation of PDGF-AA-overexpressing oligodendrocyte precursor cells promotes recovery in rat following spinal cord injury. Front. Cell. Neurosci. 11:79. doi: $10.3389 /$ fncel.2017.00079

Zheng, L., Ishii, Y., Tokunaga, A., Hamashima, T., Shen, J., Zhao, Q. L., et al. (2010). Neuroprotective effects of PDGF against oxidative stress and the signaling pathway involved. J. Neurosci. Res. 88, 1273-1284. doi: 10.1002/jnr. 22302

Zheng, L. S., Ishii, Y., Zhao, Q. L., Kondo, T., and Sasahara, M. (2013). PDGF suppresses oxidative stress induced $\mathrm{Ca} 2+$ overload and calpain activation in neurons. Oxid. Med. Cell. Longev. 2013:367206. doi: 10.1155/2013/ 367206

Conflict of Interest Statement: The authors declare that the research was conducted in the absence of any commercial or financial relationships that could be construed as a potential conflict of interest.

Copyright (c) 2019 Guo, Duan, Chen, Wang, Wang, Shen, Qi, Jiang, Zhu, Xi, Lü and $H u$. This is an open-access article distributed under the terms of the Creative Commons Attribution License (CC BY). The use, distribution or reproduction in other forums is permitted, provided the original author(s) and the copyright owner(s) are credited and that the original publication in this journal is cited, in accordance with accepted academic practice. No use, distribution or reproduction is permitted which does not comply with these terms. 